\title{
Immunological aspects of nephrotic syndrome in northern Nigeria
}

\author{
M B ABDURRAHMAN, B M GREENWOOD, P NARAYANA, F A BABAOYE, \\ AND G M EDINGTON
}

\author{
Department of Paediatrics, Department of Immunology, and Department of Pathology, \\ Ahmadu Bello University, Zaria, Nigeria
}

SUMMARY Immunological aspects of 40 northern Nigerian children with nephrotic syndrome of recent onset are reported. Eight out of 30 had hepatitis-associated antigen in their sera. Hypocomplementaemia was rare. Measurement of serum C3, C4, and ASOT was not of diagnostic value. Proteinuria selectivity index was poor in half of the patients, and appeared to depend on the severity of the kidney lesion. Abnormal immunofluorescence of kidney glomeruli to immunoglobulins, complement, Plasmodium malariae, and Plasmodium falciparum was found in 26 of the 29 children. The pattern of immunofluorescence was chiefly granular and was confined to the glomeruli. IgM was predominant. It was concluded that immunological reaction is involved in the pathogenesis of nephrotic syndrome in northern Nigerian children.

There is ample evidence that the pattern of nephrotic syndrome in tropical countries is different from that in temperate ones. Some of the characteristics of the disease peculiar to the tropics are-an older age of onset, the rarity of minimal change lesions, an association with quartan malaria, poor selectivity of proteinuria, and a poor response to corticosteroids. ${ }^{1-6}$

We have studied the immunological aspects of the syndrome in children attending this hospital. The study was approved by the Ethical Committee of the Faculty of Medicine, Ahmadu Bello University.

\section{Patients and methods}

Forty children who presented consecutively with nephrotic syndrome were studied. They had had no previous treatment. A diagnosis of the nephrotic syndrome was made in a patient if he (or she) presented with oedema and had had proteinuria of $\geqslant 2 \mathrm{~g} / 24$ hours, and had hypoalbuminaemia $<25 / \mathrm{g} / 1$.

Routine investigations were made, but in addition repeated searches (3-6) were made for malarial parasites in peripheral blood smears before administration of chloroquine, and serum ASOT, C3, and C4 levels were determined. Sera were tested for HAA, urine protein selectivity was determined, and a kidney biopsy was performed.

Peripheral blood smears were examined for malarial parasites and sera were tested for HAA in another group of children; these children were matched for age and sex but they did not suffer from renal disease. Serum ASOT was determined with Wellcome Streptolysin-O commercial kit; C3 and C4 were determined by radial immunodiffusion using monospecific antisera, and the results were recorded as a percentage of a pooled adult Nigerian plasma standard. Sera were tested for HAA by counter-current immunoelectrophoresis. To find out the proteinuria selectivity index (SI), serum and urinary IgG and albumin concentrations were determined by radial immunodiffusion using monospecific antisera. The value of SI was calculated from the formula

Urine IgG/serum IgG $\div$ urine albumin $/$ serum albumin

and expressed as a percentage. SI was classified as high $(1-15 \%)$, moderate $(16-30 \%)$, or poor $(\geqslant 31 \%){ }^{7}$
Abbreviations:
ASOT antistreptolysin $O$ titre
HAA hepatitis-associated antigen
MPGN membranoproliferative glomerulo- nephritis
PGN proliferative glomerulonephritis
SI proteinuria selectivity index
QMN quartan malaria nephropathy 


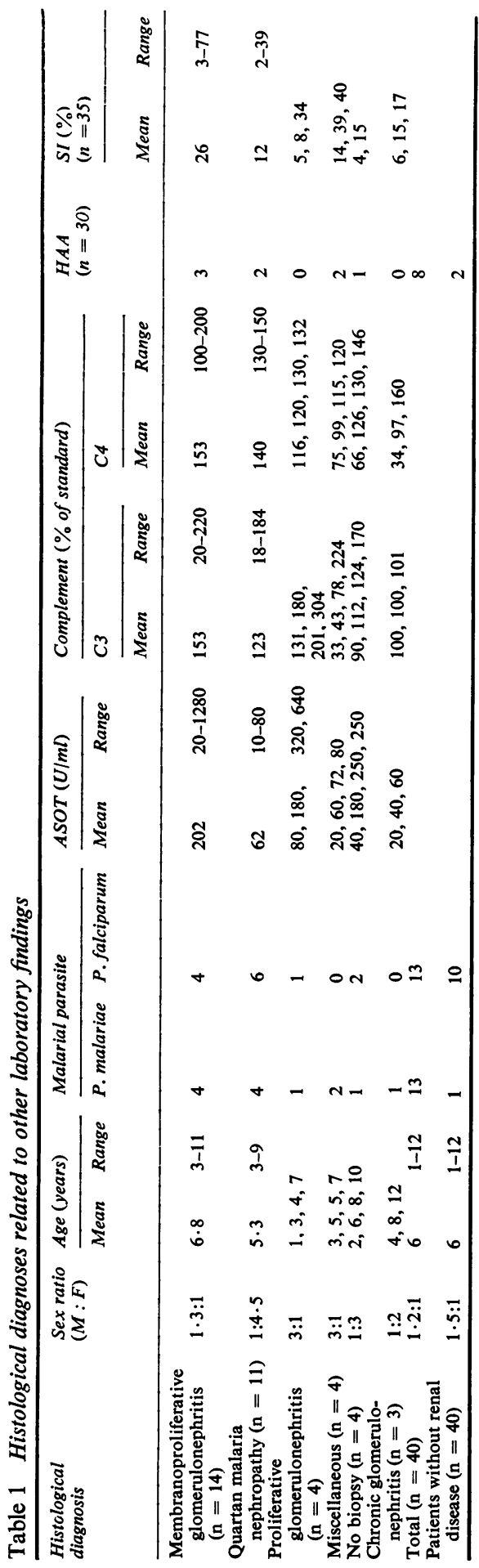

For the immunofluorescence studies renal biopsies were snap-frozen using carbon dioxide and then kept at $-20^{\circ} \mathrm{C}$ until they could be examined. Sections $6 \mu \mathrm{m}$ thick were washed for 30 minutes in phosphate-buffered saline $\mathrm{pH} 7 \cdot 2$, and then stained with the following conjugates at optimal concentrations: anti-whole immunoglobulin, anti-IgG, anti-IgA, and anti-IgM (Burroughs Wellcome), an anti-C3 conjugate prepared from Hyland antiserum, and anti-Plasmodium falciparum and antiPlasmodium malariae conjugates that had been prepared from specific antisera provided by D A Voller. Sections were examined with a Zeiss fluorescent microscope using a BG 12 primary filter and a secondary filter at 440 ängström units. The immunofluorescence was done 'blind', without knowledge of the results of the light microscopical examination of the kidney biopsies. No fluorescence was observed with any of the conjugates in 2 normal kidneys obtained from children killed in a road accident. Histological examination and classification of the kidney biopsies on light microscopy were done by one of us (G M E), without knowing the other laboratory findings.

\section{Results}

Children were divided into 6 groups according to the appearance of their kidney biopsies in the light microscopical examination: MPGN $\mathrm{n}=14$, $\mathrm{QMN} n=11, \mathrm{PGN} \mathrm{n}=4$, miscellaneous $\mathrm{n}=4$, no biopsy $n=4$, and chronic glomerulonephritis $n=3$. The 'no biopsy' group comprised 4 children in whom kidney biopsy was either not done or had been unsuccessful. The miscellaneous group comprised one child with rapidly progressive glomerulonephritis, one with congenital tubular defect, one with membranous glomerulonephritis, and one child in whom the biopsy could not be classified.

The histological diagnoses related to other laboratory findings are shown in Tables 1 and 2.

Malarial parasitaemia. The incidence of $\boldsymbol{P}$. falciparum was not greatly different in children with nephrotic syndrome compared with children without renal disease. However, more patients with nephrotic syndrome had $P$. malariae in their blood smears than patients without renal disease $(P<0.0001)$, or healthy children in the local population (P 0.01).

ASOT, C3, and C4. In each histological group, there was a wide scatter in the distribution of ASOT, C3, and $C 4$ values (Table 1), and such measurements had no diagnostic value in the individual patient. As a group, patients with QMN had the lowest levels of 
Table 2 Histological diagnoses related to immunofluorescence

\begin{tabular}{|c|c|c|c|c|c|c|c|c|}
\hline \multirow[t]{2}{*}{ Histological diagnosis } & \multicolumn{7}{|c|}{ No with immunofluorescence } & \multirow{2}{*}{$\begin{array}{l}\text { Total no of patients } \\
\text { with abnormal } \\
\text { immunofluorescence }\end{array}$} \\
\hline & Whole & $\operatorname{Ig} G$ & Ig.4 & IgM & C3 & P. falciparum & P. malariae & \\
\hline $\begin{array}{l}\text { Membranoproliferative } \\
\text { glomerulonephritis }(n=13)\end{array}$ & 8 & 3 & 3 & 10 & 10 & 2 & 2 & $12 *$ \\
\hline $\begin{array}{l}\text { Quartan malaria } \\
\text { nephropathy }(n=9) \\
\text { Proliferative }\end{array}$ & 6 & 2 & 4 & 7 & 4 & $\mathbf{0}$ & 5 & 7 \\
\hline glomerulonephritis $(\mathrm{n}=3)$ & 3 & 2 & 0 & 2 & 2 & $\mathbf{0}$ & 0 & 3 \\
\hline $\begin{array}{l}\text { Miscellaneous }(n=2) \\
\text { Chronic glomerulonephritis }\end{array}$ & 2 & 2 & $\mathbf{0}$ & 2 & 1 & $\mathbf{0}$ & $\mathbf{0}$ & 2 \\
\hline $\begin{array}{l}(n=2) \\
\text { Total }(n=29)\end{array}$ & $\begin{array}{r}2 \\
21\end{array}$ & $\begin{array}{r}1 \\
10\end{array}$ & $\begin{array}{l}0 \\
7\end{array}$ & $\begin{array}{r}2 \\
23\end{array}$ & $\begin{array}{r}1 \\
18\end{array}$ & $\begin{array}{l}1 \\
3\end{array}$ & $\begin{array}{l}1 \\
8\end{array}$ & $\begin{array}{r}2 \\
26\end{array}$ \\
\hline
\end{tabular}

* Kidney tissue from 2 patients reacted only to anti-whole serum and to no other antiserum.

ASOT (mean 62 units/ml), and patients with PGN had the highest mean value of ASOT (305 units/ml). Two patients with MPGN had high ASOT levels320 and 1280 units/ml. The fairly high C3 and C4 levels found were similar to the values in children in the local population. ${ }^{8}$

HAA. HAA was detected in 8 out of 30 sera of children with nephrotic syndrome, more often than the 2 out of 40 sera of children without renal disease $(\mathrm{P}<0 \cdot 01)$.

Proteinuria selectivity index. SI was determined in 35 patients: 19 patients had high, 9 had moderate, and 7 had poor selectivity. There was a wide range of values in each histological group. The degree of selectivity appeared to bear an inverse relationship to the severity of renal disease, but there was no relationship to age, sex, duration of symptoms, or initial laboratory findings.

Immunofluorescence. Abnormal fluorescence was found in 26 out of 29 biopsies. Immunofluorescence was predominantly granular $(80 \%)$; it was linear in $5 \%$, and mixed granular and linear in $15 \%$. The fluorescence was confined to the glomeruli except for 2 patients in whom there was additional fluorescence in the tubules. Fluorescence was most intense with IgM. Seven patients showed positive IgA fluorescence.

\section{Discussion}

In this study abnormal immunofluorescence was observed in $26(90 \%)$ out of 29 kidney biopsies. This finding suggests that nephrotic syndrome in nearly all our patients was the result of immunological damage from immune complexes. A similar finding was reported from southern Nigeria, ${ }^{9}$ east Africa, ${ }^{10}$ and South Africa. ${ }^{11}$ The pattern of immunofluorescence in different parts of Africa is similar except for IgA fluorescence. In children showing immunofluorescence the number with IgA fluorescence was 3 out of 10 in South Africa, ${ }^{11} 3$ out of 26 in east Africa, ${ }^{10} 7$ out of 26 in the present study, and none out of 41 children in southern Nigeria. ${ }^{9}$ We cannot account for these differences. In other continents the majority of cases of childhood nephrotic syndrome does not show abnormal immunofluorescence of kidney tissues. ${ }^{12}$

The evidence for immunological damage as demonstrated by immunofluorescence is supported by the finding of abnormal renal histology and poorly selective proteinuria. All the 36 renal biopsies showed pathological changes of varying severity; no biopsy showed minimal change lesions. $46 \%$ of the patients had poorly selective proteinuria. In other parts of Africa, pathological changes in kidney biopsies $^{9}$ and poor $\mathrm{SI}^{6}$ have been reported. The findings in Africa are different from those in Europe and America, where a majority of cases of childhood nephrotic syndrome had minimal lesion on light microscopical examination of kidney biopsy ${ }^{13}$ associated with highly selective proteinuria in at least $90 \%$ of cases. ${ }^{514}$

Which antigens are involved in the immunological damage to the kidney? In the present study, $P$. malariae appeared to be one, as shown by (1) the high rate of $P$. malariae parasitaemia, (2) histology of the kidney biopsies, and (3) detection of $P$. malariae antigen in the glomeruli. The prevalence of $P$. malariae parasitaemia in children with nephrotic syndrome was higher than in patients without renal disease, or in children in the local population. The histology of kidney biopsy in 11 patients is compatible with the histology of QMN described by Edington and Gilles. ${ }^{15}$

$P$. malariae antigen was detected in the glomeruli of 5 of 9 patients with QMN, 2 of 13 patients with MPGN, and 1 of 2 patients with chronic glomerulonephritis. The 2 children in the MPGN group with $P$. malariae antigen in their kidney biopsies also 
had $P$. malariae parasitaemia, and it is possible that they should have been diagnosed as having QMN.

About three-quarters of patients had evidence of immunological damage although $P$. malariae could not be demonstrated as the cause of the damage.

P. falciparum antigen was detected in the kidneys of 3 patients, all of whom had $P$. malariae antigen too. This could have been a cross-reaction with $P$. malariae antigen, although Berger et al. ${ }^{16}$ reported 3 cases of $P$. falciparum malaria associated with nephrotic syndrome who had renal histology similar to that seen in poststreptococcal acute glomerulonephritis. The histology of 4 biopsies in the present study was proliferative glomerulonephritis, associated with high ASOT levels in 3 of the children. The high levels of ASOT found in 2 patients with MPGN could have been an indication of recent infection with the streptococcus. Only 2 of the children with PGN and high ASOT values had histories that suggested recent poststreptococcal nephritis, but their clinical features were no different from the other children with the nephrotic syndrome. The role of anti-streptococcal antigens in the pathogenesis of nephrotic syndrome in our patients appears slight.

Abnormal kidney immunofluorescence has been described in patients with hepatosplenic Manson's schistosomiasis with or without evidence of renal disease. ${ }^{17-18}$ Schistomiasis, both haematobium and Manson's, occurs in Nigeria. We have not studied the role of schistosomiasis in the pathogenesis of nephrotic syndrome.

Eight $(27 \%)$ out of the 30 sera tested had HAA. This figure is higher than for children without renal disease, and it is higher too than the figure of $6.3 \%$ for healthy children aged between 2 and 5 years in the local population. ${ }^{19}$ HAA has been detected by immunofluorescence in the glomeruli of patients with nephrotic syndrome associated with hepatitisassociated antigenaemia. ${ }^{20}$ Because of the high prevalence of HAA in the nephrotic syndrome found in this study, we plan to study the role of the antigen in the aetiology of nephrotic syndrome, using anti-HAA conjugates.

We thank Mr Moses Damisa for technical assistance.

M B A was supported by a grant from Ahmadu Bello University, and B M G by a grant from the Medical Research Council of the United Kingdom.

\section{References}

1 Hendrickse R G, Gilles H M. The nephrotic syndrome and other renal diseases in children in Western Nigeria. East Afr Med J 1963; 40: 186-201.
${ }^{2}$ Kibukamusoke J W, Hutt M S R, Wilks N E. The nephrotic syndrome in Uganda and its association with quartan malaria. $Q J$ Med 1967; 36: 393-408.

3 White R H R, Glasgow E F, Mills R T. Clinicopathological study of nephrotic syndrome in childhood. Lancet $1970 ; \mathbf{i}: 1353-9$.

4 Powell K C, Meadows R. The nephrotic syndrome in New Guinea: a clinical and histological spectrum. Aust NZ J Med 1971; 1: 363-73.

5 Arneil G C. 164 children with nephrosis. Lancet 1961; ii: 1103-10.

- Adeniyi A, Hendrickse R G, Houba V. Selectivity of proteinuria and response to prednisolone or immunosuppressive drugs in children with malarial nephrosis. Lancet 1970; i: 644-8.

7 Adeniyi A, Hendrickse R G, Soothill J F. Differential protein clearances and response to treatment in Nigerian nephrotic children. Arch Dis Child 1976; 51 : 691-6.

8 Greenwood B M, Brueton M J. Complement activation in children with acute malaria. Clin Exp Immunol 1974; 18: $267-72$.

9 Hendrickse R G, Adeniyi A, Edington G M, Glasgow E F, White R H R, Houba V. Quartan malarial nephrotic syndrome. Collaborative clinicopathological study in Nigerian children. Lancet 1972; i: 1143-9.

10 Ward P A, Kibukamusoke J W. Evidence for soluble immune complexes in the pathogenesis of the glomerulonephritis of quartan malaria. Lancet 1969; i: 283-5.

11 Adhikari M, Coovadia M M, Loening W E K. The nephrotic syndrome in children. $S$ Afr Med J 1976; 50: 39-43.

12 Michael A F, Mclean R H, Rou L P, et al. Immunologic aspects of the nephrotic syndrome. Kidney Int 1973; 3: 105-15.

13 Churg J, Habib R, White, R H R. Pathology of the nephrotic syndrome in children. A report for the International Study of Kidney Disease in Children. Lancet 1970; i: 1299-302.

14 Cameron J S. Histology, protein clearances, and response to treatment in the nephrotic syndrome. Br Med J 1968; iv: $352-6$.

15 Edington G M, Gilles H M. Pathology in the tropics, second edition. London: Arnold, 1976: 631-3.

16 Berger M, Birch L M, Conte N F. The nephrotic syndrome secondary to acute glomerulonephritis during falciparum malaria. Ann Intern Med 1967; 67: 1163-71.

17 Hoshino-Shimizu S, de Brito T, Kanamura H Y, et al. Human schistosomiasis: Schistosoma mansoni antigen detection in renal glomeruli. Trans $R$ Soc Trop Med Hyg 1976; 70: 492-6.

18 Madwar M A, Voller A. Circulating soluble antigens and antibody in schistosomiasis. Br Med J 1975; i: 435-6.

19 Williams A O, Fabiyi A, Williams A I O, Gupta B, O'Connor E H, Greenwood B M. Hepatitis- $\beta$ antigen in Nigerian children. East Afr Med J 1973; 50: 521-9.

20 Takekoshi Y, Tanaka M, Miyakawa Y, Yoshizawa $\mathbf{H}$, Takahashi K, Mayumi $M$. Free 'small' and IgGassociated 'large' hepatitis $B$ e antigen in the serum and glomerular capillary walls of two patients with membranous glomerulonephritis. $N$ Engl J Med 1979; 300: 814-9.

Correspondence to Dr M B Abdurrahman, Department of Paediatrics, ABU Hospital, PMB 1026, Zaria, Kaduna State, Nigeria.

Received 23 October 1979 